\title{
Ferrofluid Flow in Magnetic Field Above Stretching Sheet with Suction and Injection
}

\section{Gabriella Bognár and Krisztián Hriczó}

\author{
University of Miskolc \\ 3515 Miskolc-Egyetemváros, Hungary \\ E-mail(corresp.): v.bognar.gabriella@uni-miskolc.hu
}

Received July 30, 2019; revised May 19, 2020; accepted May 19, 2020

\begin{abstract}
The aim of this paper is to investigate the boundary layer of ferrofluid flow induced by a permeable stretching sheet. Fluid is electrically non-conducting in the presence of non-uniform magnetic field. The governing non-linear partial differential equations are reduced to non-linear ordinary differential equations by applying a similarity transformation. Numerical solutions are obtained by using Maple. The effects of the magnetic field, the Reynolds number and the porosity on the velocity and thermal fields are investigated. The impact of the parameters on the skin friction and the local Nusselt number is numerically examined. The skin friction and heat transfer coefficients are decreasing with enhancing the stretching, the values of porosity and the ferromagnetic parameter.
\end{abstract}

Keywords: stretching sheet, suction and injection, magnetic field, ferrofluid, self-similar solution.

AMS Subject Classification: 76D05; 34A34; 35G45.

\section{Introduction}

The steady, laminar boundary layer flows driven by moving boundaries are among the classical problems of theoretical fluid mechanics (see Schlichting [30]). The investigation of fluid flow problem due to stretched surface has become important due to its application in engineering science and industrial processes, especially in metallurgical processes, drawing of plastics and elastic sheets, metal and polymer extrusion, wire drawing, hot rolling, glass fibre production, manufacturing of paper production, rubber sheet etc. [13].

The phenomenon of momentum transfer in viscous fluid flow past a steady sheet is a basic problem of laminar boundary layer flow, which was first investigated by Blasius [5] for uniform free stream using similarity transformations. 
Results on the steady laminar boundary layer flow on a moving plate in a quiescent liquid have been obtained by Sakiadis [28,29] and he gave solutions in closed and approximate forms. Crane [12] examined the flow due to the stretching of polymer sheets in the polymer industry and obtained an analytical solution to the laminar boundary layer equations. Cranes' problem was extended to heat and mass transfer with the effect of suction or blowing by Gupta and Gupta [14]. The flow and heat transfer in a saturated porous medium over a stretching surface has been examined by Vajravelu [34]. Ariel [3] has considered the problem of boundary layer flow of a viscous fluid by stretching sheet using the homotopy perturbation method.

Recently, Al-Housseiny [1] has investigated the laminar boundary layer flow due to the motion of stretching sheets by taking into account both the fluid motion as well as the motion of the sheet.

Magnetohydrodynamic flow of an incompressible viscous fluid caused by moving plane surface has been analyzed by Pavlov [26]. The heat and mass transfer over a stretching surface was examined by Cortell [11] and taking into consideration it in the saturated porous medium by Vajravelu [33], with the effects of radiation and heat source by Siddheshwar et al. [31] and with mass transpiration through porous sheet by Singh et al. [32].

Different solution techniques are applied to obtain analytic or numeric solutions to these problems. Analytic solutions to the boundary layer problem over a stretching surface were provided in [6] and [18]. The similarity method was applied in [7]. For solving MHD boundary-layer equations the modified decomposition method and Padé approximants were used in [15] and the modified differential transform method in [27].

Ferrofluid is a special type of nanofluid that contains suspended nano particles of non-magnetic carrier liquid, typically water, ethylene glycol, glycerol or oil, containing very fine magnetized particles, for example magnetite, with diameters of order 5-15 nm [25]. Ferrofluids are applied to enhancing the heat transfer rate in numerous cases in the industry. This fluid behaves like a normal fluid except that it experiences a force due to magnetization.

Neuringer [23] has investigated the saturated ferrofluids under the influence of both thermal and magnetic fields. Andersson and Valnes [2] extended the Cranes' problem for a viscous non-conducting ferrofluid. The effect of the magnetic field due to a magnetic dipole on a shear driven motion (flow over a stretching sheet) was considered and they concluded that the magnetic field decelerate the fluid motion as compared to the hydrodynamic case. Zeeshan et al. [35] investigated the effects of magnetic dipole and thermal radiation on the flow of ferromagnetic fluid on a stretching sheet. Majeed et al. [19] examined the case of ferrofluid flow over a stretching sheet along with applied magnetic field submerge with dipole effect. It was shown by Andersson [2] that an external magnetic field has the same effect on the flow as the viscoelasticity.

In the recent years, Nadeem et al. [20,21,22] presented the heat transfer and flow of boundary layer problem for MHD stagnation point flow of viscous nanofluids. Li et al. [17] and Khan et al. [16] evaluated the heat transfer and flow behavior for the stagnation point flow of nanofluids.

Regarding the above literature, we are interested in examining the magneto- 
thermomechanical interaction between heated viscous incompressible ferrofluid above a nonlinearly stretching sheet in the presence of a spatially varying magnetic field. In this paper, the stream velocity and the heat transfer in ferromagnetic viscoelastic flow past a stretching sheet with the effect of suction and injection are investigated. The impact of significant physical parameters like stretching parameter, ferromagnetic parameter, suction/injection parameter and Prandtl number is examined and presented graphically on the velocity and temperature profile.

\section{Mathematical formulation}

We apply local similarity transformations for the boundary layer flow through a permeable surface. The porous medium is bounded by a semi-infinite horizontal plate (on $x$-axis). The free stream is quiescent and the temperature far away from the plate is a power function of variable $x$.

Consider a steady two-dimensional flow of an incompressible, viscous and electrically non-conducting ferromagnetic fluid over a flat sheet placed in a horizontal direction.

\subsection{Magnetic dipole}

The ferrofluid is subjected to an external magnetizing field $\mathbf{H}$, the magnetic dipoles or line currents in the material will align and create a magnetization $\mathbf{M}$. The dipole of the magnet is placed at a distance $a$ from the surface in both sides, in such a way that its center lies on $y$-axis. The magnetic field $(\mathbf{H})$ due to the magnetic dipole is directed towards positive $x$-direction.

The ferrofluid flow is influenced by the dipole field whose magnetic scalar potential can be written as $[8,9,10]$

$$
\phi(x, y)=-\frac{I_{0}}{2 \pi}\left(\tan ^{-1} \frac{y+a}{x}+\tan ^{-1} \frac{y-a}{x}\right)
$$

where $I_{0}$ denotes the dipole moment per unit length and $a$ is the distance of the line currents from the leading edge. The wall temperature is a decreasing function of $x$ and is given by $T_{w}=T_{c}+A x^{\alpha}$, where $T_{c}$ denotes the Curie temperature, $A$ and $\alpha$ are real constants.

The negative gradient of the magnetic scalar potential $\phi$ equals the applied magnetic field, i.e. $\mathbf{H}=-\nabla \phi$.

The components $H_{x}$ and $H_{y}$ of magnetic field intensity along the coordinates $x$ and $y$ axes are

$$
\begin{aligned}
& H_{x}=-\frac{\partial \phi}{\partial x}=-\frac{I_{0}}{2 \pi}\left[\frac{y+a}{x^{2}+(y+a)^{2}}+\frac{y-a}{x^{2}+(y-a)^{2}}\right] \\
& H_{y}=-\frac{\partial \phi}{\partial y}=-\frac{I_{0}}{2 \pi}\left[\frac{x}{x^{2}+(y+a)^{2}}+\frac{x}{x^{2}+(y-a)^{2}}\right] .
\end{aligned}
$$


The second derivatives are

$$
\frac{\partial^{2} \phi}{\partial x^{2}}=-\frac{\partial^{2} \phi}{\partial y^{2}}=-\frac{I_{0}}{2 \pi}\left[\frac{2 x(y+a)}{\left[x^{2}+(y+a)^{2}\right]^{2}}+\frac{2 x(y-a)}{\left[x^{2}+(y-a)^{2}\right]^{2}}\right]
$$

and

$$
\frac{\partial^{2} \phi}{\partial x \partial y}=-\frac{I_{0}}{2 \pi}\left[\frac{(y+a)^{2}-x^{2}}{\left[x^{2}+(y+a)^{2}\right]^{2}}+\frac{(y-a)^{2}-x^{2}}{\left[x^{2}+(y-a)^{2}\right]^{2}}\right] .
$$

In ferrohydrodynamic interactions, the existence of spatially varying fields is required [24]. For the exposition of ferrohydrodynamic interaction we shall use the following assumptions:

(i) the fluid temperature must be less than Curie temperature,

(ii) the applied magnetic field is inhomogeneous.

Applying the scalar potential $\phi, \nabla H$ is calculated as follows

$$
\nabla H=\left([\nabla H]_{x},[\nabla H]_{y}\right)=\left(\frac{\frac{\partial \phi}{\partial x} \frac{\partial^{2} \phi}{\partial x^{2}}+\frac{\partial \phi}{\partial y} \frac{\partial^{2} \phi}{\partial x \partial y}}{\sqrt{\left(\frac{\partial \phi}{\partial x}\right)^{2}+\left(\frac{\partial \phi}{\partial y}\right)^{2}}}, \frac{\frac{\partial \phi}{\partial x} \frac{\partial^{2} \phi}{\partial x \partial y}+\frac{\partial \phi}{\partial y} \frac{\partial^{2} \phi}{\partial y^{2}}}{\sqrt{\left(\frac{\partial \phi}{\partial x}\right)^{2}+\left(\frac{\partial \phi}{\partial y}\right)^{2}}}\right),
$$

where $[\nabla H]_{x}$ and $[\nabla H]_{y}$ denote the first and second components of $\nabla H$, respectively.

Since $(\partial \phi / \partial x)_{y=0}=0$ and $\left(\partial^{2} \phi / \partial y^{2}\right)_{y=0}=0$ at the wall, then $[\nabla H]_{y}$ vanishes.

In the boundary layer for regions close to the wall when distances from the leading edge large compared to the distances of the line sources from the plate, i.e. $x \gg a$, then one gets

$$
[\nabla H]_{x}=-\frac{I_{0}}{\pi} \frac{1}{x^{2}}
$$

The variation of magnetization $M$ is assumed as a linear function of temperature $M=K\left(T_{c}-T\right)$, where $K$ is the pyromagnetic coefficient (see [2]).

Once the ferromagnetic fluid approaches Curie temperature, furthermore, there is no magnetization.

\subsection{Flow analysis}

The stretching of the sheet induces a fluid velocity field $(u(x, y), v(x, y))$ which satisfies the equations governing the flow [35]

$$
\begin{aligned}
\frac{\partial u}{\partial x}+\frac{\partial v}{\partial y} & =0 \\
u \frac{\partial u}{\partial x}+v \frac{\partial u}{\partial y} & =-\frac{I_{o} \mu_{0} K}{\pi \rho}\left(T_{c}-T\right) \frac{1}{x^{2}}+\nu \frac{\partial^{2} u}{\partial y^{2}}, \\
c\left[u \frac{\partial T}{\partial x}+v \frac{\partial T}{\partial y}\right] & =k \frac{\partial^{2} T}{\partial y^{2}},
\end{aligned}
$$


where the $x$ and $y$ axes are taken parallel and perpendicular to the plate, $u$ and $v$ are the parallel and normal velocity components to the plate, respectively, $\mu_{0}$ means the permeability of the vacuum, $\nu$ is the kinematic viscosity and $\rho$ denotes the density of the ambient a fluid, which will be assumed constant. The appropriate boundary conditions to the problem are at the surface $(y=0)$ :

$$
u(x, 0)=u_{w}, \quad v(x, 0)=V(x), \quad T(x, 0)=T_{w}
$$

with $T_{w}=T_{c}+A x^{\alpha}$ and

$$
u(x, y) \rightarrow u_{\infty}, \quad T(x, y) \rightarrow T_{\infty}
$$

as $y$ leaves the boundary layer $(y \rightarrow \infty)$ with $T_{\infty}=T_{c}$, and $u_{w}$ is the stretching speed which is assumed throughout the paper to be $u_{w}=U_{w} x^{m}$ and $u_{\infty}=0$. Parameter $\alpha$ is relating to the power law exponent. The value $\alpha=1$ refers to a linear temperature profile and $m=0$ for constant exterior streaming speed. In case of $\alpha=2$, the temperature profile is quadratic and for $m=1$ the streaming speed is linear. The value of $\alpha=0$ corresponds to no temperature variation on the surface.

The sheet is permeable and subjected to suction velocity $(0 ; V(x))$ (see [30], Ch. 11, pp. 302), where $V(x)=-V_{0} \sqrt{\frac{m+1}{2}}\left(\frac{x}{L}\right)^{\frac{m-1}{2}}, m>-1$. As a convention, $V(x)>0$ implies suction while $V(x)<0$ implies injection of the fluid at $y=0$.

Introducing the stream function $\psi$, defined by $u=\partial \psi / \partial y$ and $v=-\partial \psi / \partial x$, problem (2.1)-(2.3) can be formulated as

$$
\begin{aligned}
& \frac{\partial \psi}{\partial y} \frac{\partial^{2} \psi}{\partial y \partial x}-\frac{\partial \psi}{\partial x} \frac{\partial^{2} \psi}{\partial y^{2}}=\nu \frac{\partial^{3} \psi}{\partial y^{3}}-\frac{I_{0} \mu_{0} K}{\pi \rho}\left(T_{c}-T\right), \\
& c\left[\frac{\partial \psi}{\partial y} \frac{\partial T}{\partial x}-\frac{\partial \psi}{\partial x} \frac{\partial T}{\partial y}\right]=k \frac{\partial^{2} T}{\partial y^{2}} .
\end{aligned}
$$

Boundary conditions (2.4) and (2.5) are transformed to

$$
\begin{aligned}
\frac{\partial}{\partial y} \psi(x, 0) & =U_{w} x^{m}, & \frac{\partial}{\partial x} \psi(x, 0) & =V(x), & T(x, 0) & =T_{c}+A x^{\alpha}, \\
\frac{\partial}{\partial y} \psi(x, y) & \rightarrow 0, & T(x, y) & =T_{c} & \text { as } y & \rightarrow \infty .
\end{aligned}
$$

We look for similarity solutions of a class of solutions $\psi$ and $T$ in the form (see [4])

$$
\psi(x, y)=C_{1} x^{\kappa} f(\eta), \quad T=T_{c}+A x^{\alpha} \Theta(\eta), \quad \eta=C_{2} x^{\tau} y,
$$

where $\kappa$ and $\tau$ satisfy the scaling relation $\kappa+\tau=m$ and for positive coefficients $C_{1}$ and $C_{2}$ the relation $C_{1} / C_{2}=\nu$. The real numbers $\kappa$ and $\tau$ are such that $\kappa-\tau=1$ and $C_{1} C_{2}=U_{\infty}$, hence $\kappa=(\alpha-1) / 4, \quad \tau=(\alpha-3) / 4, \quad C_{1}=$ $\sqrt{\nu U_{\infty} / \lambda}, \quad C_{2}=\sqrt{U_{\infty} / \nu \lambda}$.

Then, we get the following similarity system of ordinary differential equations for $\alpha=2 m+1$ :

$$
f^{\prime \prime \prime}-(\alpha-1)\left(f^{\prime}\right)^{2}+\frac{\alpha+1}{4} f f^{\prime}-\frac{\beta}{\lambda^{2}} \Theta=0
$$




$$
\Theta^{\prime \prime}+\operatorname{Pr}\left(\frac{\alpha+1}{4} f \Theta^{\prime}-\alpha \Theta f^{\prime}\right)=0,
$$

where prime denotes differentiation with respect to $\eta$. The boundary conditions for the similarity functions $f$ and $\theta$ are

$$
\begin{aligned}
& f(0)=V_{c}, \quad f^{\prime}(0)=\lambda, \quad \Theta(0)=1, \\
& f^{\prime}(\eta)=0, \quad \Theta(\eta)=0 \quad \text { as } \eta \rightarrow \infty,
\end{aligned}
$$

where $\operatorname{Pr}=c \nu / k$ is the Prandtl number, $\beta=I_{0} \mu_{0} K A /\left(\pi \rho U_{w}^{2}\right), \lambda=U_{w} /\left(C_{1} C_{2}\right)$ and the dimensionless measure of suction/injection known as the mass transpiration parameter is $V_{c}=V_{0} L \sqrt{R e / v}$ with Reynolds number $R e=U_{w} L / v$.

The components of the non-dimensional velocity $\mathbf{v}=(u, v)$ can be expressed by

$$
\begin{aligned}
u & =U_{w} x^{m} f^{\prime}(\eta), \\
v & =\frac{V(x)}{V_{c}}\left(\frac{m+1}{2} f(\eta)+\frac{m-1}{2} f^{\prime}(\eta) \eta\right) .
\end{aligned}
$$

Skin friction and local Nusselt number are defined as $C_{f}=\frac{2 \tau_{w}}{\rho U_{w}^{2}}$ and $N u_{x}=$ $\frac{x q_{w}}{T_{w}-T_{\infty}}$ and

$$
\tau_{y=0}=\nu \rho\left(\frac{\partial u}{\partial y}\right)_{y=0} \text { and } q_{w}=-k\left(\frac{\partial T}{\partial y}\right)_{y=0},
$$

where $\tau_{w} \sim f^{\prime \prime}(0)$ and $q_{w} \sim \Theta^{\prime}(0)$ and $f^{\prime \prime}(0)$ denotes the skin friction coefficient and $\Theta^{\prime}(0)$ stands for the heat transfer coefficient [33].

We note that for $m=0$ and $\beta=0$, equation (2.6) is equivalent to the Blasius equation for laminar boundary-layer problem of Newtonian fluids (see [4]):

$$
f^{\prime \prime \prime}+\frac{1}{2} f f^{\prime}=0
$$

The coupled boundary-layer equations for $m=0$ were first examined for $u_{w}=0$ and $u_{\infty}=$ const. in [23].

\section{Numerical results with suction or injection}

The coupled ordinary differential equations (2.6)-(2.7) are highly non-linear. Exact analytical solutions are not possible for the complete set of transformed equations subject to the boundary conditions (2.8)-(2.9). Hence, we apply a numerical method for the solution process.

The self-similar non-linear differential equations (2.6)-(2.7) subjected to the boundary conditions (2.8)-(2.9) are solved numerically using BVP solution technique built in Maple. The boundary conditions are considered in such a way that the far field boundary conditions are satisfied at a finite value, say $\eta_{\max }$. The impact of physical parameters, namely the ferromagnetic parameter $\beta$, suction parameter $V_{c}$, power exponent $m$ and Prandtl number Pr on boundary 


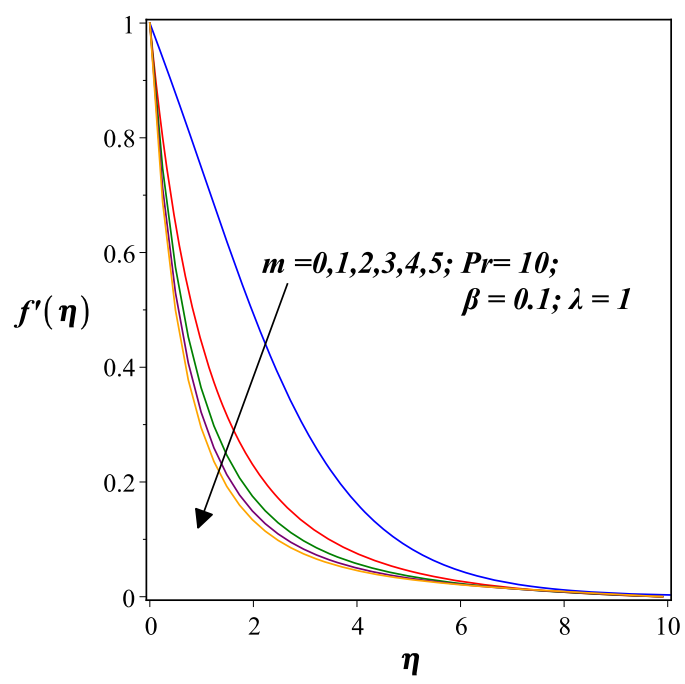

Figure 1. Effect of parameter $m$ on the dimensionless velocity along horizontal wall for $V_{c}=-1$.

layer flow and heat transfer in ferrofluid, are examined numerically. The value $\eta_{\max }$ for numerical purpose is taken as $\eta_{\max }=10$.

The ferromagnetic parameter $\beta$ highlights the effect of the external magnetic field. We want to study the parametric dependence of the boundary layer flow. Figure 1 illustrates the effect of the power-law parameter $m$ on the horizontal velocity profile $f^{\prime}(\eta)$ for the set of parameters: the Prandtl number $\operatorname{Pr}=10$ and the values of $\beta=0.1 \lambda=1$ and $V_{c}=-1$. With increasing stretching power parameter the fluid flow velocity is decreasing, the flow slows down.

We define the boundary layer thickness to be $h(x)$, such that as $y \rightarrow h(x)$, $\partial u / \partial y \rightarrow 0$ and for $y>h(x), u=0$. It is also observed that the boundary layer thickness $h(x)$ is decreasing with increasing value of $m$. Similarly, the temperature shows also decreasing tendency for increasing the power-law $m$ with the same set of parameters (see Figures 2-3). However, the thermal boundary layer thickness is smaller than the hydrodynamic boundary layer thickness $h(x)$.

The effect of various physical parameters including ferromagnetic interaction parameter $\beta$, Prandtl number $\operatorname{Pr}$ and suction/injection parameter $V_{c}$ on the temperature profiles is investigated. Figures 4 and 5 represent the influence of suction/injection parameter $V_{c}$ on velocity and on temperature profiles. Both the velocity and the temperature profiles depreciate with increasing suction parameter $V_{c}$. Figures 6-11 represent the influence of suction/injection parameter $V_{c}$ on velocity and temperature profiles for both suction and injection. The dashed curve always belongs to the case no suction or injection, $V_{c}=0$.

The skin friction coefficient $C_{f}$ is negative. In the case of injection $\left(V_{c}>0\right)$ the decrease of $f^{\prime \prime}(0)$ is greater than for suction with increasing value of either that the $m$ or $\beta$. 


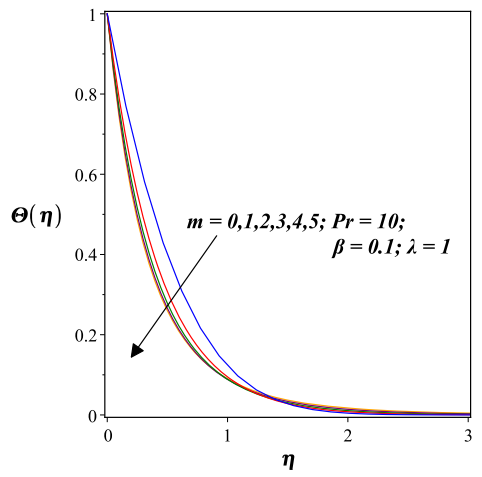

Figure 2. Effect of stretching power $m$ on the temperature for $V_{c}=-1$.

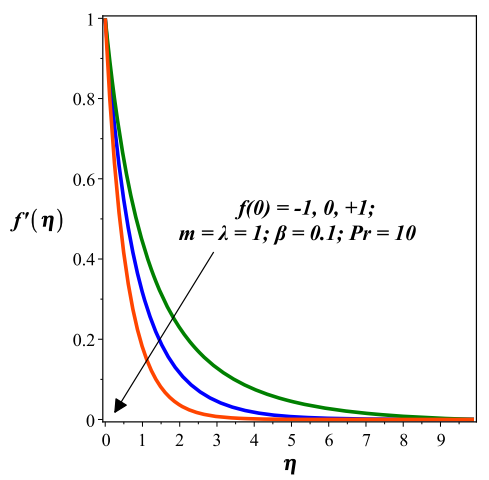

Figure 4. Effect of suction parameter $V_{c}$ on the velocity distribution.

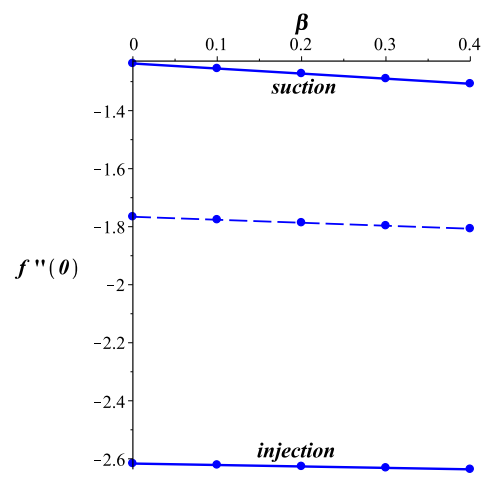

Figure 6. Effect of ferromagnetic interaction parameter $\beta$ on the skin friction coefficient.

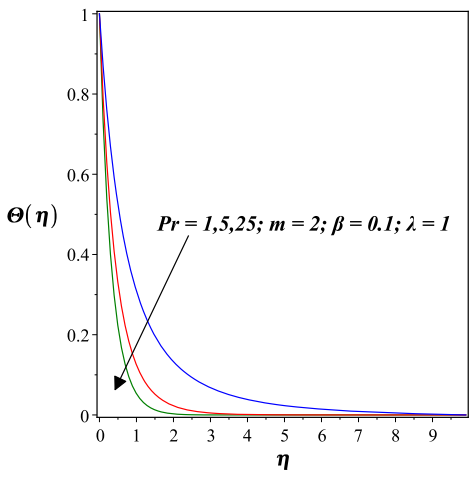

Figure 3. Effect of Prandtl number $\operatorname{Pr}$ on the temperature for $V_{c}=-1$.

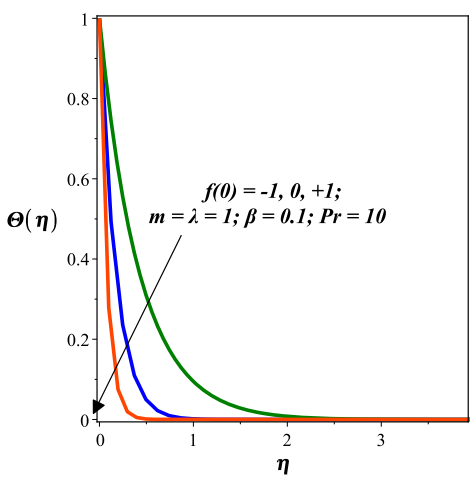

Figure 5. Effect of suction parameter $V_{c}$ on the temperature distribution.

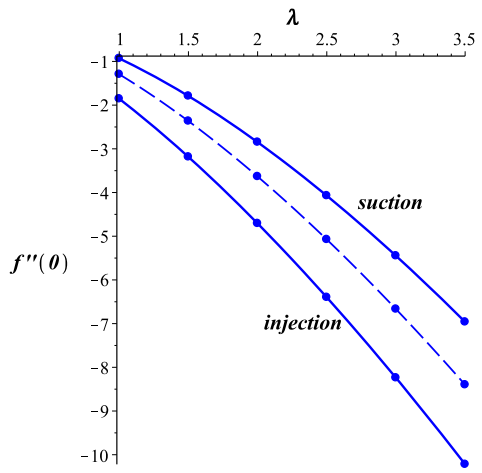

Figure 7. Effect of parameter $\lambda$ on the skin friction coefficient. 


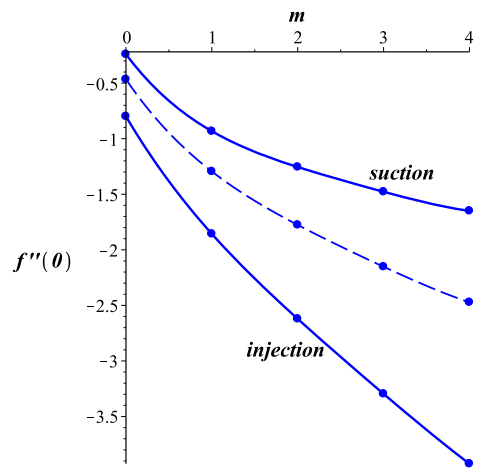

Figure 8. Effect of power-law exponent $m$ on the skin friction coefficient.

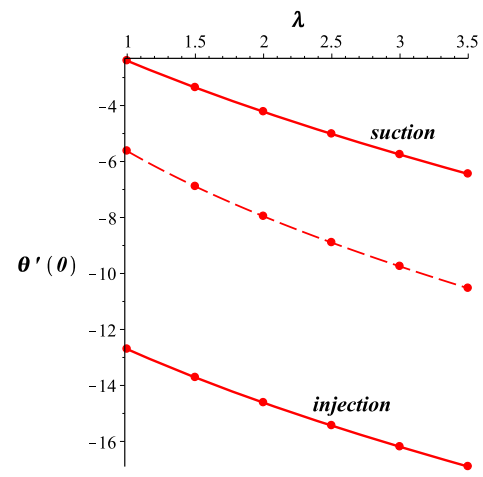

Figure 10. Effect of parameter $\lambda$ on the heat transfer coefficient.

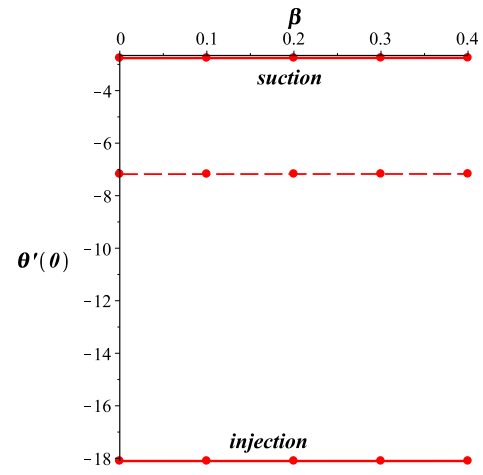

Figure 9. Effect of ferromagnetic parameter $\beta$ on the heat transfer coefficient.

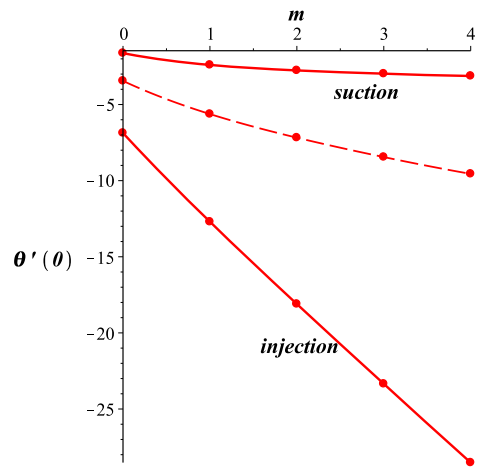

Figure 11. Effect of power-law exponent $m$ on the heat transfer coefficient.

It can be seen that temperature profile depreciates with suction parameter $\left(V_{c}=-1\right)$ and with injection $\left(V_{c}=1\right)$, but its degree is significantly different (see Figures 9-11). The values of $\theta^{\prime}(0)$ are smaller for injection than for suction.

\section{Conclusions}

The purpose of this paper was to investigate the two-dimensional ferrofluid flow and heat transfer of ferrofluid past a nonlinearly stretching sheet with suction/injection with the effect of a magnetic dipole. The governing partial differential equations of motion and heat transfer are transformed into nonlinear coupled ordinary differential equations using appropriate similarity transformations. Numerical computations are carried out to obtain the results for the boundary layer flow and heat transfer induced by a horizontal stretching sheet in magnetic field. 
Values of the skin friction and the local Nusselt number for different values of the physical parameters were investigated. The influence of the applied external magnetic field due to magnetic dipole is demonstrated through ferromagnetic interaction parameter $\beta$ in the skin friction and heat transfer coefficients. The impact of the parameters are introduced on figures.

\section{Acknowledgements}

This work was supported by Project no. 129257 implemented with the support provided from the National Research, Development and Innovation Fund of Hungary, financed under the $K_{-} 18$ funding scheme.

\section{References}

[1] T.T. Al-Housseiny and H.A. Stone. On boundary-layer flows induced by the motion of stretching surfaces. Journal of Fluid Mechanics, 706:597-606, 2012. https://doi.org/10.1017/jfm.2012.292.

[2] H.I. Andersson. MHD flow of a viscoelastic fluid past a stretching surface. Acta Mechanica, 95(1):227-230, 1992. https://doi.org/10.1007/BF01170814.

[3] P.D. Ariel. MHD flow of a viscoelastic fluid past a stretching sheet with suction. Acta Mechanica, 105(1):49-56, 1994. https://doi.org/10.1007/BF01183941.

[4] G.I. Barenblatt. Scaling, Self-similarity, and Intermediate Asymptotic. Cambridge University Press, Cambridge, 1996. https://doi.org/10.1017/CBO9781107050242.

[5] H. Blasius. Grenzschichten in Flüssigkeiten mit kleiner Reibung. InauguralDissertation ... von H. Blasius, ... Druck von B.G. Teubner, 1907. Available from Internet: https://books.google.lt/books?id=VDV3QwAACAAJ.

[6] G. Bognár. Analytic solutions to the boundary layer problem over a stretching wall. Computers $\&$ Mathematics with Applications, 61(8):2256-2261, 2011. https://doi.org/10.1016/j.camwa.2010.09.039.

[7] G. Bognár. On similarity solutions of boundary layer problems with upstream moving wall in non-Newtonian power-law fluids. IMA Journal of Applied Mathematics, 77(4):546-562, 2011. https://doi.org/10.1093/imamat/hxr033.

[8] G. Bognár and K. Hriczó. Ferrofluid flow along stretched surface under the action of magnetic dipole. WSEAS Transactions on Heat and Mass Transfer, 13:103-108, 2018.

[9] G. Bognár and K. Hriczó. Similarity transformation approach for a heated ferrofluid flow in the presence of magnetic field. Electronic Journal of Qualitative Theory of Differential Equations, 2018(42):1-15, 2018. https://doi.org/10.14232/ejqtde.2018.1.42.

[10] G. Bognár and K. Hriczó. Ferrofluid flow in the presence of magnetic dipole. Technische Mechanik, 39(1):3-15, 2019.

[11] R. Cortell. A note on flow and heat transfer of a viscoelastic fluid over a stretching sheet. International Journal of Non-Linear Mechanics, 41(1):78-85, 2006. https://doi.org/10.1016/j.ijnonlinmec.2005.04.008.

[12] L.J. Crane. Flow past a stretching plate. Zeitschrift für angewandte Mathematik und Physik ZAMP, 21(4):645-647, 1970. https://doi.org/10.1007/BF01587695. 
[13] E.G. Fisher. Extrusion of plastics. John Wiley \& Sons, 1976.

[14] P.S. Gupta and A.S. Gupta. Heat and mass transfer on a stretching sheet with suction or blowing. The Canadian Journal of Chemical Engineering, 55(6):744746, 1977. https://doi.org/10.1002/cjce.5450550619.

[15] T. Hayat, Q. Hussain and T. Javed. The modified decomposition method and Padé approximants for the MHD flow over a non-linear stretching sheet. Nonlinear Analysis: Real World Applications, 10(2):966-973, 2009. https://doi.org/10.1016/j.nonrwa.2007.11.020.

[16] A.U. Khan, S. Nadeem and S.T. Hussain. Phase flow study of MHD nanofluid with slip effects on oscillatory oblique stagnation point flow in view of inclined magnetic field. Journal of Molecular Liquids, 224:1210-1219, 2016. https://doi.org/10.1016/j.molliq.2016.10.102.

[17] X. Li, A.U. Khan, M.R. Khan, S. Nadeem and S.U. Khan. Oblique stagnation point flow of nanofluids over stretching/shrinking sheet with CattaneoChristov heat flux model: Existence of dual solution. Symmetry, 11(9):1070, 2019. https://doi.org/10.3390/sym11091070.

[18] S.-J. Liao. On the analytic solution of magnetohydrodynamic flows of nonNewtonian fluids over a stretching sheet. Journal of Fluid Mechanics, 488:189212, 2003. https://doi.org/10.1017/S0022112003004865.

[19] A. Majeed, A. Zeeshan, S.Z. Alamri and R. Ellahi. Heat transfer analysis in ferromagnetic viscoelastic fluid flow over a stretching sheet with suction. Neural Computing and Applications, 30(6):1947-1955, 2018. https://doi.org/10.1007/s00521-016-2830-6.

[20] S. Nadeem, M.R. Khan and A.U. Khan. MHD oblique stagnation point flow of nanofluid over an oscillatory stretchingshrinking sheet: Existence of dual solutions. Physica Scripta, 94(7):075204, 2019. https://doi.org/10.1088/14024896/ab0973.

[21] S. Nadeem, M.R. Khan and A.U. Khan. MHD stagnation point flow of viscous nanofluid over a curved surface. Physica Scripta, 94(11):115207, 2019. https://doi.org/10.1088/1402-4896/ab1eb6.

[22] S. Nadeem, N. Ullah, A.U. Khan and T. Akbar. Effect of homogeneousheterogeneous reactions on ferrofluid in the presence of magnetic dipole along a stretching cylinder. Results in Physics, 7:3574-3582, 2017. https://doi.org/10.1016/j.rinp.2017.09.006.

[23] J.L. Neuringer. Some viscous flows of a saturated ferro-fluid under the combined influence of thermal and magnetic field gradients. International Journal of Non-Linear Mechanics, 1(2):123-137, 1966. https://doi.org/10.1016/00207462(66)90025-4.

[24] J.L. Neuringer and R.E. Rosensweig. Ferrohydrodynamics. The Physics of Fluids, 7(12):1927-1937, 1964. https://doi.org/10.1063/1.1711103.

[25] S.S. Papell. Low viscosity magnetic fluid obtained by the colloidal suspension of magnetic particles, 1965. US Patent 3,215,572

[26] K.B. Pavlov. Magnetohydrodynamic flow of an incompressible viscous fluid caused by deformation of a plane surface. Magnitnaya Gidrodinamika, 10(4):146147, 1974. https://doi.org/10.22364/mhd. (in Russian)

[27] M.M. Rashidi. The modified differential transform method for solving MHD boundary-layer equations. Computer Physics Communications, 180(11):22102217, 2009. https://doi.org/10.1016/j.cpc.2009.06.029. 
[28] B.C. Sakiadis. Boundary-layer behavior on continuous solid surfaces: I. Boundary-layer equations for two-dimensional and axisymmetric flow. AIChE Journal, 7(1):26-28, 1961. https://doi.org/10.1002/aic.690070108.

[29] B.C. Sakiadis. Boundary-layer behavior on continuous solid surfaces: II. The boundary layer on a continuous flat surface. AIChE journal, 7(2):221-225, 1961. https://doi.org/10.1002/aic.690070211.

[30] H. Schlichting and K. Gersten. Boundary-layer theory. Springer, Berlin, 2016. https://doi.org/10.1007/978-3-662-52919-5.

[31] P.G. Siddheshwar and U.S. Mahabaleswar. Effects of radiation and heat source on MHD flow of a viscoelastic liquid and heat transfer over a stretching sheet. International Journal of Non-Linear Mechanics, 40(6):807-820, 2005. https://doi.org/10.1016/j.ijnonlinmec.2004.04.006.

[32] J. Singh, U.S. Mahabaleshwar and G. Bognár. Mass transpiration in nonlinear MHD flow due to porous stretching sheet. Scientific Reports, 9(1):18484, 2019. https://doi.org/10.1038/s41598-019-52597-5.

[33] K. Vajravelu. Flow and heat transfer in a saturated porous medium over a stretching surface. ZAMM-Journal of Applied Mathematics and Mechanics/Zeitschrift für Angewandte Mathematik und Mechanik, 74(12):605-614, 1994. https://doi.org/10.1002/zamm.19940741209.

[34] K. Vajravelu and J.R. Cannon. Fluid flow over a nonlinearly stretching sheet. Applied Mathematics and Computation, 181(1):609-618, 2006. https://doi.org/10.1016/j.amc.2005.08.051.

[35] A. Zeeshan, A. Majeed and R. Ellahi. Effect of magnetic dipole on viscous ferrofluid past a stretching surface with thermal radiation. Journal of Molecular Liquids, 215:549-554, 2016. https://doi.org/10.1016/j.molliq.2015.12.110. 